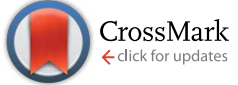

Cite this: RSC Adv., 2017, 7, 11543

\title{
Effects of Fe doping on the thermal hysteresis of the $\mathrm{La}_{0.5} \mathrm{Ca}_{0.5} \mathrm{MnO}_{3}$ system
}

\begin{abstract}
Ankam Bhaskar, M.-S. Huang and Chia-Jyi Liu*
A series of polycrystalline $\mathrm{La}_{0.5} \mathrm{Ca}_{0.5} \mathrm{Mn}_{1-x} \mathrm{Fe}_{x} \mathrm{O}_{3}(x=0.010,0.025,0.050,0.075,0.100,0.125,0.150,0.175$ and 0.200 ) was synthesized using a solid state reaction. We investigated the electrical resistivity, thermopower, and magnetization as a function of temperature. $\mathrm{La}_{0.5} \mathrm{Ca}_{0.5} \mathrm{MnO}_{3}$ exhibits a large thermal hysteresis in its electrical resistivity, thermopower, and magnetization, which can be attributed to the charge density waves pinned by impurities. The thermal hysteresis decreases with increasing Fe content up to $x=0.050$ and disappears at even higher $x$. $\mathrm{La}_{0.5} \mathrm{Ca}_{0.5} \mathrm{MnO}_{3}$ shows nonmetal-like behavior in terms of its electrical resistivity within the entire investigated temperature range of $80-300 \mathrm{~K}$, while the $x=$ 0.010 and 0.025 samples show metal-nonmetal transitions in their electrical resistivity at about 137$149 \mathrm{~K}$. The metal-nonmetal transition can be attributed to the reduction of charge ordering at small Fe content values. However, there is no metal-nonmetal transition observed for $x \geq 0.050$, which arises from the suppression of the double exchange mechanism at high Fe content values. The activation energy derived from electrical resistivity differs from that derived from thermopower, indicating that the conduction mechanism is polaronic transport in $\mathrm{La}_{0.5} \mathrm{Ca}_{0.5} \mathrm{Mn}_{1-x} \mathrm{Fe}_{x} \mathrm{O}_{3}$. The magnetic transition temperature is observed at $\sim 168 \mathrm{~K}$ and $\sim 135 \mathrm{~K}$ for $x=0.010$ and 0.025 , respectively. There is no magnetic transition observed for $x=0.100$ and 0.200 .
\end{abstract}

Received 9th December 2016 Accepted 6th February 2017

DOI: 10.1039/c6ra27974k

rsc.li/rsc-advances increasing replacement level. Their transport properties are modified as well. ${ }^{13-15}$ Moreover, the $\mathrm{La}_{0.5} \mathrm{Ca}_{0.5} \mathrm{MnO}_{3}$ system with a ratio of $\mathrm{Mn}^{3+} / \mathrm{Mn}^{4+}=1$ exhibits thermal hysteresis in its electrical resistivity and magnetic properties., ${ }^{\mathbf{1 , 2 , 1 2}}$ Therefore, it is interesting to investigate the effects of partial replacement of $\mathrm{Mn}$ with $\mathrm{Fe}$ on the thermal hysteresis of the $\mathrm{La}_{0.5} \mathrm{Ca}_{0.5} \mathrm{MnO}_{3}$ system. To the best of our knowledge, there is no systematic examination of the electrical resistivity, thermopower, and magnetic properties of a wide range of replacement levels of $\mathrm{Fe}$ in $\mathrm{La}_{0.5} \mathrm{Ca}_{0.5} \mathrm{MnO}_{3}$. In addition, there is no report regarding the thermopower and transport properties of $\mathrm{La}_{0.5} \mathrm{Ca}_{0.5^{-}}$ $\mathrm{Mn}_{1-x} \mathrm{Fe}_{x} \mathrm{O}_{3}$. In this paper, we present a systematic investigation of the electrical resistivity, thermopower, and magnetic properties of $\mathrm{La}_{0.5} \mathrm{Ca}_{0.5} \mathrm{Mn}_{1-x} \mathrm{Fe}_{x} \mathrm{O}_{3}(x=0.010,0.025,0.050$, $0.075,0.100,0.125,0.150,0.175$ and 0.200$)$. The electrical resistivity, thermopower, and magnetic measurements exhibit a strong thermal hysteresis for the undoped sample in a measurement cycle of cooling and warming. The width of hysteresis decreases with increasing Fe content up to $x=$ 0.025 , which might be related to the disappearance of the charge density waves pinned by impurities. There is no transition observed for $x>0.075$. For higher doping levels of Fe, all the samples obey the small polaron hopping mechanism. The ferromagnetic transition temperature is $168 \mathrm{~K}$ and $135 \mathrm{~K}$ for $x=0.010$ and $x=0.025$, respectively. There is no magnetic transition observed for $x=0.100$ and 0.200 . 


\section{Experimental details}

$\mathrm{La}_{0.5} \mathrm{Ca}_{0.5} \mathrm{Mn}_{1-x} \mathrm{Fe}_{x} \mathrm{O}_{3}(x=0.025,0.050,0.075,0.100,0.125$, $0.150,0.175$, and 0.200 ) ceramics were synthesized by quantitatively mixing high purity powders of $\mathrm{La}_{2} \mathrm{O}_{3}, \mathrm{CaO}, \mathrm{Fe}_{2} \mathrm{O}_{3}$ and $\mathrm{Mn}_{2} \mathrm{O}_{3}$. The mixed powders were ground using a Retsch Model MM 2000 Laboratory Mixer Mill and then calcined at $1300{ }^{\circ} \mathrm{C}$ for $18 \mathrm{~h}$ with intermediate grinding. The powders were pressed into a parallelepiped measuring $12.7 \mathrm{~mm}$ long, $2.45 \mathrm{~mm}$ wide, and $1.1 \mathrm{~mm}$ thick, followed by sintering at $1300{ }^{\circ} \mathrm{C}$ in oxygen for $18 \mathrm{~h}$. Powder X-ray diffraction patterns were obtained using a Scintag DMS 2000 diffractometer equipped with a $\mathrm{Cu} \mathrm{K} \alpha$ radiation source. Electrical resistivity as a function of temperature was measured using a standard four-probe technique. Thermopower measurements as a function of temperature were performed between $75 \mathrm{~K}$ and $300 \mathrm{~K}$ using steady-state techniques with a temperature gradient of $0.5-1 \mathrm{~K}$ across the sample. A type E differential thermocouple was used to measure the temperature difference between the hot and cold ends of the sample. ${ }^{16,17}$ A commercial superconducting quantum interference device magnetometer (Quantum Design) was used to characterize the magnetic properties of the samples.

\section{Results and discussion}

\subsection{Structural analysis}

Fig. 1a shows the X-ray diffraction patters of $\mathrm{La}_{0.5} \mathrm{Ca}_{0.5} \mathrm{Mn}_{1-x} \mathrm{Fe}_{x} \mathrm{O}_{3}$ with $x=0.025,0.050,0.075,0.100,0.125,0.150,0.175$, and 0.200 . All the samples are of a single phase. All the diffraction peaks are matched with the standard powder diffraction data (ICDD no. 46-0513), the diffraction peaks of the samples could be indexed based on an orthorhombic crystal structure with space group Pnma. The undoped $\mathrm{La}_{0.5} \mathrm{Ca}_{0.5} \mathrm{MnO}_{3}$ has lattice parameters of $a=5.42 \pm 0.02 \AA, b=7.64 \pm 0.01 \AA$, and $c=$ $5.43 \pm 0.02 \AA$. Fig. $1 \mathrm{~b}$ shows the variation of the lattice parameters with changing Fe content. The lattice parameters are almost constant with increasing $\mathrm{Fe}$ content due to the identical ionic radius sizes of $\mathrm{Fe}^{3+}$ and $\mathrm{Mn}^{3+}$ (0.645 $\mathrm{A}$ ). Similar results were also observed by Ahn et al., ${ }^{18,19}$ Jonker et al. ${ }^{20}$ and Orlova $e$ al. ${ }^{21}$ for the $\mathrm{Mn}$ site partially replaced by the Fe ions in $\mathrm{La}_{1-x} \mathrm{Ca}_{x} \mathrm{Mn}_{1-y} \mathrm{Fe}_{y} \mathrm{O}_{3}$.

\subsection{Electrical transport properties}

Fig. 2 shows the temperature dependence of the electrical resistivity for $\mathrm{La}_{0.5} \mathrm{Ca}_{0.5} \mathrm{Mn}_{1-x} \mathrm{Fe}_{x} \mathrm{O}_{3}$ with $x=0.00,0.010$, and 0.025. The electrical resistivity measurements were first carried out by cooling and then warming between $300 \mathrm{~K}$ and $80 \mathrm{~K}$. For the undoped sample, the electrical resistivity slowly increases up to $\sim 149 \mathrm{~K}$ with decreasing temperature followed by a dramatic increase upon further cooling; the electrical resistivity decreases until reaching $\sim 200 \mathrm{~K}$ upon warming, then slowly decreases with increasing temperature. On increasing/ decreasing the temperature, the electrical resistivity changes by several orders of magnitude. It should be noted that the electrical resistivity does not follow the same path during the warming and cooling cycle, and it yields a strong thermal
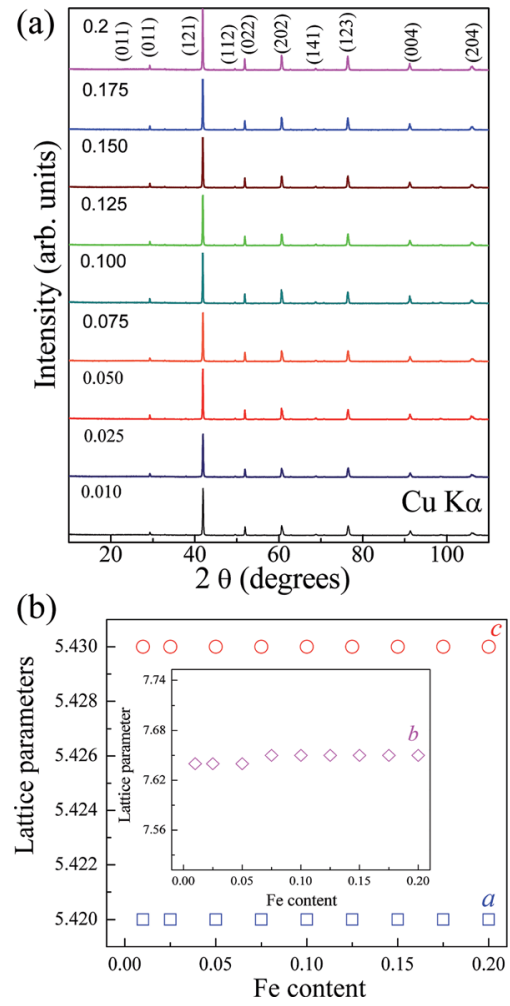

Fig. 1 (a) $\mathrm{X}$-ray diffraction patterns of $\mathrm{La}_{0.5} \mathrm{Ca}_{0.5} \mathrm{Mn}_{1-x} \mathrm{Fe}_{x} \mathrm{O}_{3}(x=$ $0.010,0.025,0.050,0.075,0.100,0.125,0.150,0.175$, and 0.200 ) and (b) lattice parameters for $\mathrm{La}_{0.5} \mathrm{Ca}_{0.5} \mathrm{Mn}_{1-x} \mathrm{Fe}_{x} \mathrm{O}_{3}$ with different $\mathrm{Fe}$ contents

hysteresis, which arises from a first-order phase transition. The electrical resistivity yields a strong hysteresis between $110 \mathrm{~K}$ and $210 \mathrm{~K}$ in the cooling and warming cycle. The first-order phase transition can be attributed to an incommensurate-tocommensurate charge-ordering transition occurring at $\sim 149 \mathrm{~K}$ and $\sim 200 \mathrm{~K}$ upon cooling and warming, respectively. ${ }^{22}$ Similar results were also observed previously. ${ }^{1,3}$ The electrical resistivity increases initially until reaching a certain temperature and then decreases with decreasing (increasing) temperature during the cooling (warming) cycle for $x=0.010,0.025$ (Fig. 2). As

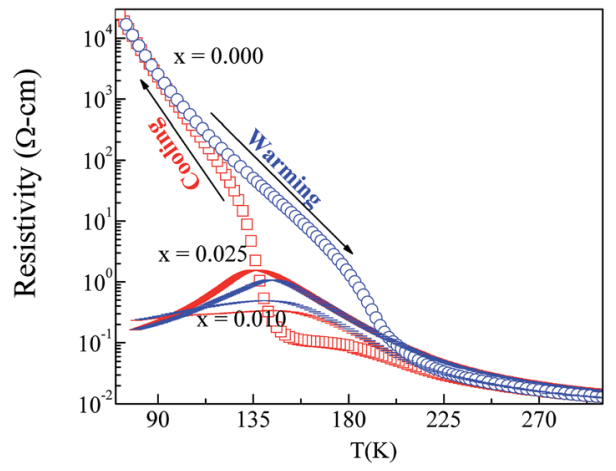

Fig. 2 Temperature dependence of the electrical resistivity for $x=$ $0.000,0.010$, and 0.025 . The blue symbols represent the sample as it is warming up and the red symbols as it is cooling down. 
compared to $\mathrm{La}_{0.5} \mathrm{Ca}_{0.5} \mathrm{MnO}_{3}$, a smaller thermal hysteresis is observed for the Fe-doped samples. The electrical resistivity at $80 \mathrm{~K}$ is $\sim 1 \times 10^{4}, \sim 0.15$, and $\sim 0.25 \Omega \mathrm{cm}$ for $\mathrm{La}_{0.5} \mathrm{Ca}_{0.5} \mathrm{MnO}_{3}$, $x=0.010$, and 0.025 , respectively. According to previous reports, ${ }^{12,18,19,23}$ the charge ordering reduces linearly with increasing Fe content in $\mathrm{La}_{1-x} \mathrm{Ca}_{x} \mathrm{Mn}_{1-y} \mathrm{Fe}_{y} \mathrm{O}_{3}$. Dhiman et al. ${ }^{23}$ reported that the electrical resistivity is reduced by nearly two orders of magnitude for $\mathrm{La}_{0.5} \mathrm{Ca}_{0.5} \mathrm{Mn}_{0.98} \mathrm{Fe}_{0.02} \mathrm{O}_{3}$ as compared to $\mathrm{La}_{0.5} \mathrm{Ca}_{0.5} \mathrm{MnO}_{3}$ due to suppression of the charge ordering state. Dhiman et al. ${ }^{23}$ also reported that the charge ordering state decreases linearly with increasing Fe content up to $x<0.06$ in $\mathrm{La}_{0.5} \mathrm{Ca}_{0.5} \mathrm{Mn}_{1-x} \mathrm{Fe}_{x} \mathrm{O}_{3}$. Ahn et al. ${ }^{18,19}$ reported that the charge ordering state is observed at $210 \mathrm{~K}$ for $\mathrm{La}_{0.47} \mathrm{Ca}_{0.53} \mathrm{Mn}_{0.91} \mathrm{Fe}_{0.09^{-}}$ $\mathrm{O}_{3}$. Chen et al. ${ }^{12}$ reported that the charge ordering state is observed at $\sim 200 \mathrm{~K}$ for $\mathrm{La}_{0.5+0.5 x} \mathrm{Ca}_{0.5-0.5 x} \mathrm{Mn}_{1-x} \mathrm{Fe}_{x} \mathrm{O}_{3}(x=0.04)$. Moreover, it can be readily seen in Fig. 2 that the metalnonmetal transition occurs at $149 \mathrm{~K}$ on warming ( $147 \mathrm{~K}$ on cooling) for $x=0.010$ and $143 \mathrm{~K}$ on warming $(\sim 137 \mathrm{~K}$ on cooling) for $x=0.025$. As $\mathrm{Fe}^{3+}$ partially replaces $\mathrm{Mn}^{3+}$, there is a competition between the antiferromagnetic superexchange and ferromagnetic double exchange interactions. As a result, the ferromagnetic double exchange interaction gradually disappears with increasing $\mathrm{Fe}^{3+}$ content, which leads to the disappearance of metal-like behavior at low temperatures. Rao et $a .^{24}$ also observed the metal-nonmetal transition in $\mathrm{La}_{0.7^{-}}$ $\mathrm{Ca}_{0.3} \mathrm{Mn}_{1-x} \mathrm{Fe}_{x} \mathrm{O}_{3}$. Furthermore, they reported that the transition temperature decreases with increasing Fe content. Fig. 3 shows the temperature dependence of the electrical resistivity for $\mathrm{La}_{0.5} \mathrm{Ca}_{0.5} \mathrm{Mn}_{1-x} \mathrm{Fe}_{x} \mathrm{O}_{3}$ with $x=0.050$. In the warming/ cooling cycle, the electrical resistivity curves of $x=0.050$ exhibit a small thermal hysteresis. Unlike the samples with a smaller Fe content ( $x=0.010$ and 0.025$)$, there is no metalnonmetal transition observed down to $80 \mathrm{~K}$ and the electrical resistivity continuously increases with decreasing temperature. Fig. 4 shows the temperature dependence of the electrical resistivity for $\mathrm{La}_{0.5} \mathrm{Ca}_{0.5} \mathrm{Mn}_{1-x} \mathrm{Fe}_{x} \mathrm{O}_{3}$ with $x=0.075,0.100,0.125$, $0.150,0.175$ and 0.200 . The electrical resistivity continuously decreases with increasing temperature, and no transition is observed for $x \geq 0.050$. It should be noted that there is no

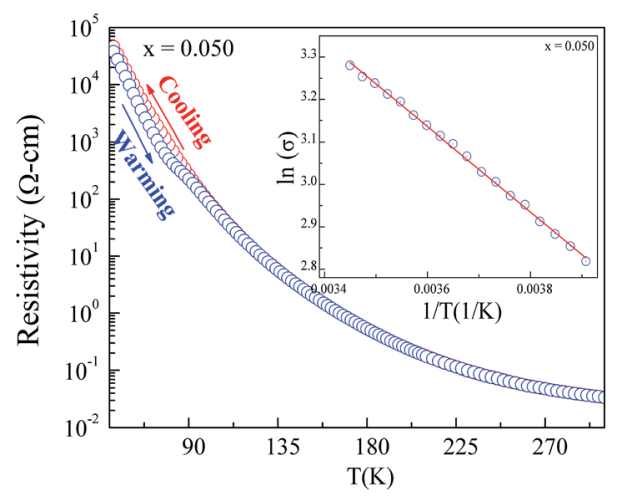

Fig. 3 Temperature dependence of the electrical resistivity for $x=$ 0.05 . Inset: In $\sigma$ vs. $1 / T$ for $x=0.05$. The blue symbols represent the sample as it is warming up and the red symbols as it is cooling down. The solid line is the linear fitting using eqn (1). thermal hysteresis observed in the cooling and warming cycle, which could be attributed to the suppression of charge ordering states. This clearly shows that these samples exhibit nonmetallike behavior throughout the entire temperature range (80-300 K). This might be associated with the number of itinerant electrons being depleted with increasing Fe content and hence a weakening of the double exchange interactions.

Now we turn to the conduction mechanism of $\mathrm{La}_{0.5} \mathrm{Ca}_{0.5^{-}}$ $\mathrm{Mn}_{1-x} \mathrm{Fe}_{x} \mathrm{O}_{3}$. The lattice of a manganite becomes distorted around the electrons in the conduction band at high temperatures and the formation of small polarons ensues due to the strong electron-phonon interactions. Thermally activated hopping of these polarons plays an important role above the magnetic transition. ${ }^{25}$ For polaronic transport, the activation energy derived from the measurements of electrical resistivity and thermopower is distinguishable. The electrical conduction for carriers activated to polaronic states can be expressed as

$$
\sigma \approx \sigma_{0} \mathrm{e}^{-\left(E_{\mathrm{g}}+E_{\mathrm{p}}\right) / 2 k_{\mathrm{B}} T},
$$

where the activation energy $E_{\sigma}=\left(E_{\mathrm{g}}+E_{\mathrm{p}}\right) / 2, E_{\mathrm{g}}$ is the energy gap for carriers being excited across, $E_{\mathrm{p}}$ is the polaron binding energy, $k_{\mathrm{B}}$ is the Boltzmann constant and $T$ is the absolute temperature. We analyze the electrical resistivity versus the temperature data using eqn (1). The activation energy for the electrical conduction can be obtained by the slope of the curve fitting of $\ln \sigma$ versus $T^{-1}$. Typical plots of $\ln \sigma$ versus $T^{-1}$ for $x=0.050$ and 0.200 are shown in the insets of Fig. 3 and 4, respectively. The derived activation energies of these samples are 115.0, 115.1, 108.9, 108.8, 107.0, 109.7, and $109.9 \mathrm{meV}$ for $x=0.050,0.075,0.100,0.125,0.150$, 0.175 , and 0.200 , respectively.

\subsection{Thermal transport properties}

Thermopower measurements are a very sensitive probe to the type and characteristic energy of carriers and are a complementary tool to the electrical resistivity measurements for transport property studies. Since thermopower is a measure of the heat per carrier over temperature, we can thus view it as a measure of the entropy per carrier. Carriers with different

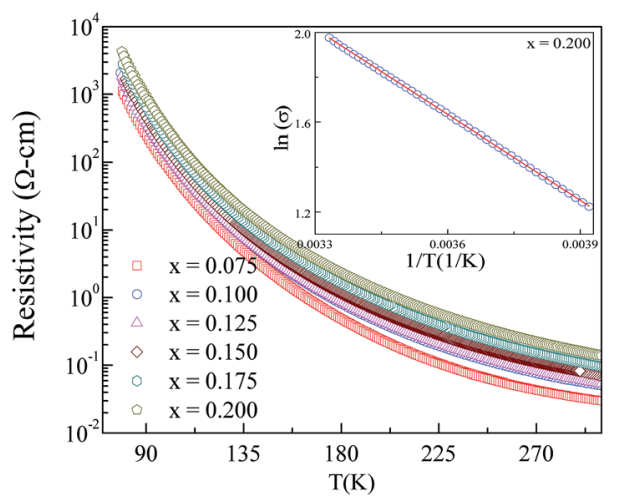

Fig. 4 Temperature dependence of the electrical resistivity for $x=$ $0.075,0.100,0.125,0.150,0.175$, and 0.200 . Inset: $\ln \sigma$ vs. $1 / T$ for $x=$ 0.200 . Solid line is the linear fitting using eqn (1). 
characteristic energies determine the temperature dependence of thermopower. Fig. 5 shows the temperature dependence of the thermopower for $\mathrm{La}_{0.5} \mathrm{Ca}_{0.5} \mathrm{Mn}_{1-x} \mathrm{Fe}_{x} \mathrm{O}_{3}$ with $x=0.000$, 0.010 and 0.025 . The sign of thermopower is negative for all the samples in the entire temperature range ( $80-300 \mathrm{~K})$, indicating that the carriers are electrons. The inset of Fig. 5 reveals that $\mathrm{La}_{0.5} \mathrm{Ca}_{0.5} \mathrm{MnO}_{3}$ exhibits a large thermopower hysteresis, which is in agreement with the hysteresis observed in the electrical resistivity. A similar thermopower hysteresis was observed in the impurity-pinned CDW in $\mathrm{Lu}_{5} \mathrm{Rh}_{4} \mathrm{Si}_{10}{ }^{26}$ Smontara et al. ${ }^{27}$ reported that a large thermopower hysteresis arises from the interaction between the inhomogeneous CDW superlattice and a quasi-periodic defect structure in the $\left(\mathrm{NbSe}_{4}\right)_{10} \mathrm{I}_{3}$ system. On initial cooling of $\mathrm{La}_{0.5} \mathrm{Ca}_{0.5} \mathrm{MnO}_{3}$, the absolute value of the thermopower is nearly constant down to $225 \mathrm{~K}$, then gradually decreases as the temperature decreases until $138 \mathrm{~K}$, followed by a rapid increase on further cooling to $110 \mathrm{~K}$. On warming, the absolute value of thermopower is nearly constant up to $90 \mathrm{~K}$, then gradually increases until $126 \mathrm{~K}$, followed by a moderate decrease on further heating to $201 \mathrm{~K}$, and then slowly decreases until $225 \mathrm{~K}$. For the $x=0.010$ sample, on cooling (warming) the absolute value of thermopower gradually increases (increases) until $\sim 177 \mathrm{~K}(\sim 179 \mathrm{~K})$, followed by a dramatic decrease (increase) on further cooling (warming) to $\sim 140 \mathrm{~K}(\sim 142 \mathrm{~K})$. For the $x=0.025$ sample, on cooling the absolute value of thermopower slowly increases (decreases) until $\sim 157 \mathrm{~K}(\sim 159 \mathrm{~K})$, followed by a dramatic decrease (increase) upon further cooling (warming) to $\sim 129 \mathrm{~K}(\sim 134 \mathrm{~K})$. It should be noticed that a smaller thermopower hysteresis is observed for $x=0.010$ and 0.025 as compared to $\mathrm{La}_{0.5} \mathrm{Ca}_{0.5} \mathrm{MnO}_{3}$. The smaller thermopower hysteresis is attributed to a decrease in the charge ordering caused by the Fe substitution. Similar behavior is observed in the resistivity measurements. Now we turn to the thermopower of $\mathrm{La}_{0.5} \mathrm{Ca}_{0.5} \mathrm{Mn}_{1-x} \mathrm{Fe}_{x} \mathrm{O}_{3}$ with higher Fe content values. Fig. 6 and 7 show the temperature dependence of thermopower for $x=0.050,0.075,0.100,0.0125,0.150,0.175$, and 0.200 . For both the $x=0.050$ and $x=0.075$ samples, the thermopower exhibits an upturn behavior (Fig. 6). It can be readily seen that in Fig. 7 that the upturn behavior shifts to higher temperatures for $x>0.075$.

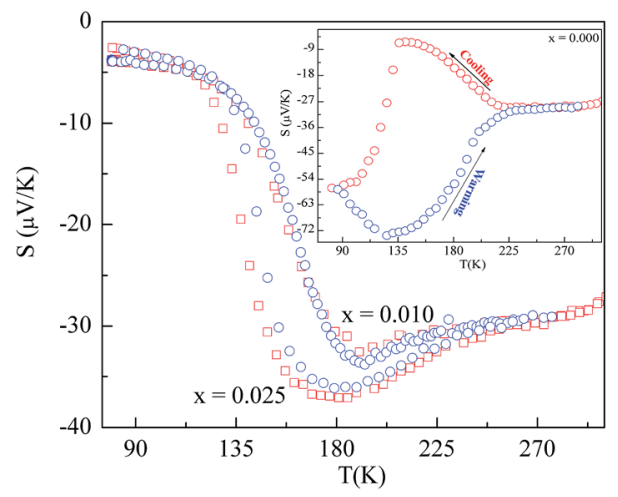

Fig. 5 Temperature dependence of thermopower for $x=0.010$, 0.025 . The blue symbol represents the sample as it is warming up and the red symbol as it is cooling down.

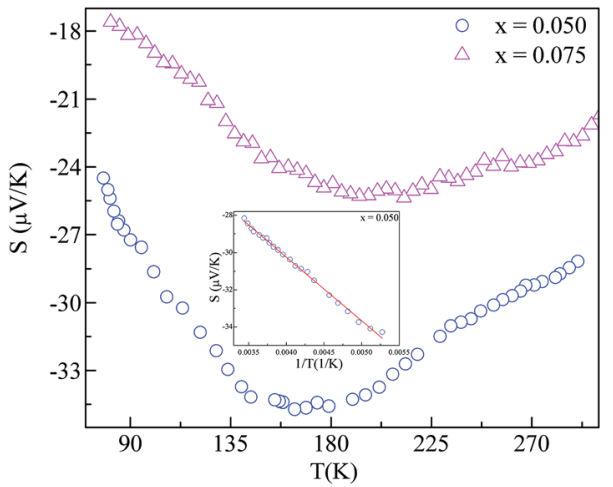

Fig. 6 Temperature dependence of thermopower for $x=0.050$, 0.075 . Inset: $S$ vs. $T^{-1}$ for $x=0.050$. Solid line is the linear fitting using eqn (2).

To facilitate the understanding of the transport mechanism, we analyze the thermopower data for carriers activated to polaronic states using eqn (2),

$$
S \approx \frac{k_{\mathrm{B}}}{e}\left(\frac{E_{\mathrm{g}}}{2 k_{\mathrm{B}} T}+B\right),
$$

where $B$ is associated with the spin and the mixing entropy ${ }^{28}$ and the activation energy $E_{\mathrm{s}}=E_{\mathrm{g}} / 2$. The activation energies $\left(E_{\mathrm{s}}\right)$ derived from the thermopower data can be obtained by the slope of the curve fitting of $S$ versus $T^{-1}$. Typical plots of $S$ versus $T^{-1}$ for $x=0.050$ and 0.100 are shown in the insets of Fig. 6 and 7 , respectively. The derived activation energies $\left(E_{\mathrm{s}}\right)$ are 3.5, 4.6, $3.0,3.3 \mathrm{meV}$ for $x=0.050,0.075,0.100$, and 0.125 , respectively. It can be readily seen that the activation energy $\left(E_{\mathrm{s}}\right)$ derived from the thermopower data differs from that derived from the resistivity data, which indicates polaronic transport. ${ }^{29-31}$

\subsection{Magnetic properties}

Fig. 8 shows the temperature dependence of the zero-fieldcooled (ZFC) and field-cooled (FC) magnetization $(M)$ of $\mathrm{La}_{0.5^{-}}$ $\mathrm{Ca}_{0.5} \mathrm{Mn}_{1-x} \mathrm{Fe}_{x} \mathrm{O}_{3}(x=0.000,0.010,0.025,0.100$, and 0.200) in an applied field of 5000 Oe. The ZFC magnetization of

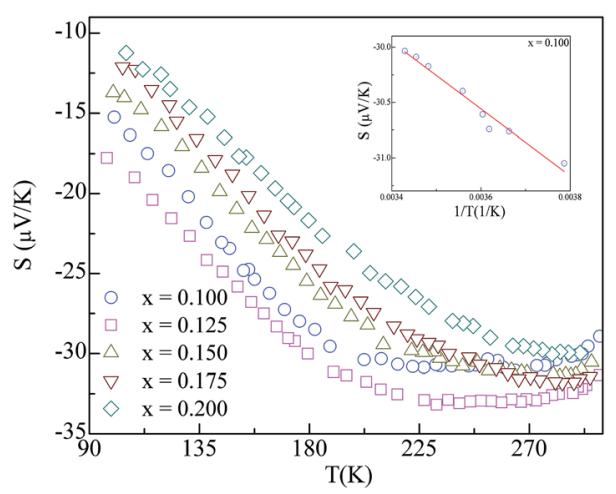

Fig. 7 Temperature dependence of thermopower for $x=0.100$, $0.125,0.150,0.175$, and 0.200 . Inset: $S$ vs. $T^{-1}$ for $x=0.100$. Solid line is the linear fitting using eqn (2). 


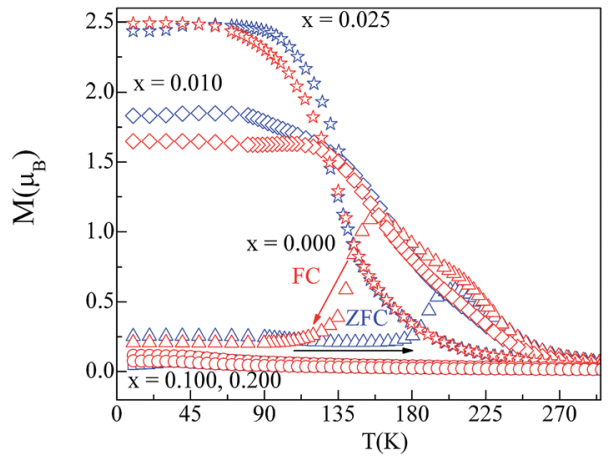

Fig. 8 Temperature dependence of zero-field-cooling (color: blue) and field-cooling (color: red) magnetization for $x=0.000,0.010$, $0.025,0.100$, and 0.200 (applied field: 5000 Oe).

$\mathrm{La}_{0.5} \mathrm{Ca}_{0.5} \mathrm{MnO}_{3}$ is almost constant until reaching $\sim 180 \mathrm{~K}\left(T_{\mathrm{N}}\right)$, and then gradually increases up to $\sim 212 \mathrm{~K}\left(\sim T_{\mathrm{C}}\right)$, followed by a decrease with increasing temperature. The FC magnetization follows the same path from room temperature to $270 \mathrm{~K}$, then gradually increases until $150 \mathrm{~K}\left(T_{\mathrm{N}}\right)$, and then decreases down to $98 \mathrm{~K}$, followed by a nearly constant value with decreasing temperature. Similar behavior was also reported previously. ${ }^{1,3,32}$ For the $x=0.010$ and 0.025 samples, the ZFC magnetization is nearly constant until reaching a certain temperature, and then decreases rapidly with increasing temperature; after that it merges with the FC magnetization curve. The value of the magnetic moment increases for $x=0.010$ and 0.025 as compared to $\mathrm{La}_{0.5} \mathrm{Ca}_{0.5} \mathrm{MnO}_{3}$. Similar results were obtained previously. ${ }^{12,33}$ The bifurcation in the ZFC-FC magnetization is observed at temperatures below $T_{\mathrm{N}}$ for $\mathrm{La}_{0.5} \mathrm{Ca}_{0.5} \mathrm{MnO}_{3}$. The magnetization of ZFC is higher than that of $\mathrm{FC}$ at temperatures below $T_{\mathrm{N}}$, which is in contrast to its general behavior. In general, at temperatures below spin-glass-like transition, the FC magnetization is larger than that of ZFC. However, our experimental results are quite different from a spin-glass-like behavior. This might arise from the fact that the ferromagnetic ordering developed below $T_{\mathrm{C}}$ is completely suppressed in the antiferromagnetic state. This indicates that there are two phases coexisting at temperatures below $T_{\mathrm{N}}$, namely, the

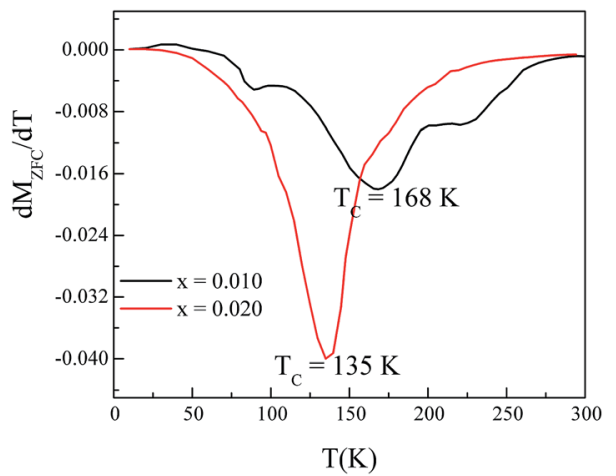

Fig. 9 Temperature dependence of the derivative of the ZFC magnetization with respect to temperature for $x=0.010$ and 0.025 . ferromagnetic and antiferromagnetic clusters. Therefore, it is conceivable that the ferromagnetic domain shrinks in size during the FC measurements, the FC magnetization is consequently lower than that of ZFC. Similar results also were reported by Das et al. ${ }^{34}$ A similar trend is observed for $x=0.010$. However, the FC magnetization is slightly larger than that of ZFC for $x=0.025$, which might be related to the coexistence of ferromagnetic and spin-glass-like states. Dhiman et al. ${ }^{23}$ also reported a spin-glass-like behavior in $\mathrm{La}_{0.5} \mathrm{Ca}_{0.5} \mathrm{Mn}_{0.08} \mathrm{Fe}_{0.02} \mathrm{O}_{3}$. Moreover, Kekade et $a l .{ }^{35}$ investigated the magnetic hysteresis $(M-H)$ loops of $\mathrm{La}_{0.5} \mathrm{Ca}_{0.5} \mathrm{MnO}_{3}$ at different temperatures. They reported the coexistence of charge-ordered antiferromagnetic and ferromagnetic states observed at $\leq 150 \mathrm{~K}$, and that a paramagnetic nature is observed at $300 \mathrm{~K}$. Dhiman et al. ${ }^{23}$ reported that a narrow hysteresis is observed due to the coexistence of ferromagnetic clusters in the charge and the orbital ordered antiferromagnetic matrix during $M-H$ measurements of $\mathrm{La}_{0.5^{-}}$ $\mathrm{Ca}_{0.5} \mathrm{Mn}_{0.99} \mathrm{Fe}_{0.01} \mathrm{O}_{3}$. Chen et al. ${ }^{12}$ conducted $M-H$ loops experiments with $\mathrm{La}_{0.5} \mathrm{Ca}_{0.5} \mathrm{Mn}_{1-x} \mathrm{Fe}_{x} \mathrm{O}_{3}$. They concluded that the saturation magnetization initially increases for $x=0.04$, then decreases for $x=0.10$ with increasing Fe content. Fig. 9 shows the inflection points of the $\mathrm{d} M_{\mathrm{ZFC}} / \mathrm{d} T$ versus temperature curves, which exhibit a minimum at $\sim 168 \mathrm{~K}$ for $x=0.010$ and $\sim 135 \mathrm{~K}$ for $x=0.025$, which correspond to the ferromagnetic transition.

\section{Conclusions}

We have investigated the temperature dependence of the electrical resistivity, thermopower, and magnetic properties of a series of $\mathrm{La}_{0.5} \mathrm{Ca}_{0.5} \mathrm{Mn}_{1-x} \mathrm{Fe}_{x} \mathrm{O}_{3}$ with $x=0.0010,0.025,0.050$, $0.075,0.100,0.125,0.150,0.175$ and 0.200 . Thermal hysteresis is observed in the electrical resistivity, thermopower, and magnetic measurements for $\mathrm{La}_{0.5} \mathrm{Ca}_{0.5} \mathrm{MnO}_{3}$ and samples with $x=0.010$ and 0.025 . The thermal hysteresis can be attributed to charge density waves pinned by impurities. The $x=0.010$ and 0.025 samples exhibit a smaller thermal hysteresis than $\mathrm{La}_{0.5^{-}}$ $\mathrm{Ca}_{0.5} \mathrm{MnO}_{3}$ and show a nonmetal-to-metal transition in their electrical resistivities. Samples with $x \geq 0.050$ exhibit a nonmetal-like behavior in the entire temperature range (80$300 \mathrm{~K})$. The magnitude of thermopower remains negative in the entire temperature range, indicating that the majority carriers are electrons. $\mathrm{La}_{0.5} \mathrm{Ca}_{0.5} \mathrm{MnO}_{3}$ shows a large thermal hysteresis in its electrical resistivity, thermopower and magnetization. The thermal hysteresis becomes smaller upon partial Mn replacement with Fe and eventually disappears when the Fe content is greater than 0.050 . The activation energy derived from electrical resistivity differs from that derived from thermopower, indicating that the conduction mechanism is polaronic transport in $\mathrm{La}_{0.5} \mathrm{Ca}_{0.5} \mathrm{Mn}_{1-x} \mathrm{Fe}_{x} \mathrm{O}_{3}$. The ferromagnetic transition is observed at $\sim 168 \mathrm{~K}$ and $\sim 135 \mathrm{~K}$ for $x=0.010$ and $x=0.025$, respectively. No magnetic transition is observed for $x=0.100$ and 0.200 .

\section{Acknowledgements}

This work was supported by the Ministry of Science and Technology of Taiwan under the Grant No. 104-2112-M-018-002- 
MY3. Ankam Bhaskar would like to express thanks to the visiting scientist fellowship sponsored by MOST of Taiwan.

\section{References}

1 P. G. Radaelli, D. E. Cox, M. Marezio and S.-W. Cheong, Charge, orbital, and magnetic ordering in $\mathrm{La}_{0.5} \mathrm{Ca}_{0.5} \mathrm{MnO}_{3}$, Phys. Rev. B: Condens. Matter Mater. Phys., 1997, 55, 30153023.

2 S.-W. Cheong, H. Y. Hwang, C. H. Chen, B. Batlogg, L. W. Rupp Jr and S. A. Carter, Charge-ordered states in (La,Sr) ${ }_{2} \mathrm{NiO}_{4}$ for hole concentration $n_{\mathrm{h}}=1 / 3$ and $1 / 2$, Phys. Rev. B: Condens. Matter Mater. Phys., 1994, 49, 7088-7091.

3 P. Schiffer, A. P. Ramirez, W. Bao and S.-W. Cheong, Low temperature magnetoresistance and the magnetic phase diagram of $\mathrm{La}_{1-x} \mathrm{Ca}_{x} \mathrm{MnO}_{3}$, Phys. Rev. Lett., 1995, 75, 33363339.

4 M. Rubinstein, D. J. Gillespie, J. E. Snyder and T. M. Tritt, Effect of $\mathrm{Gd}$, Co, and $\mathrm{Ni}$ doping in $\mathrm{La}_{2 / 3} \mathrm{Ca}_{1 / 3} \mathrm{MnO}_{3}$ : resistivity, thermopower, and paramagnetic resonance, Phys. Rev. B: Condens. Matter Mater. Phys., 1997, 56, 54125423.

5 M.-H. Phan, N. D. Tho, N. Chan, S.-C. Yu and M. Kurisu, Large magnetic entropy change above $300 \mathrm{~K}$ in a colossal magnetoresistive material $\mathrm{La}_{0.7} \mathrm{Sr}_{0.3} \mathrm{Mn}_{0.98} \mathrm{Ni}_{0.02} \mathrm{O}_{3}$, J. Appl. Phys., 2005, 97, 103901.

6 E. Banks and N. Tashima, Magnetically ordered pervoskites in the system $\mathrm{La}_{1-x} \mathrm{Ca}_{x} \mathrm{Mn}_{1-x} \mathrm{Fe}_{x} \mathrm{O}_{3}, J$. Appl. Phys., 1970, 41, 1186-1189.

7 K. De, R. Ray, R. N. Panda, S. Giri, H. Nakumura and T. Kohara, The effect of Fe substitution on magnetic and transport properties of $\mathrm{LaMnO}_{3}$, J. Magn. Magn. Mater., 2005, 288, 339-346.

8 T. R. N. Kutty and J. Philip, Electrical transport properties and magnetoresistance of $\mathrm{LaMn}_{1-x} \mathrm{M}_{x} \mathrm{O}_{3+\delta}$ substituted with diamagnetic ions $(\mathrm{M}=\mathrm{Li}, \mathrm{Mg}, \mathrm{Ti}), J$. Phys.: Condens. Matter, 2000, 12, 7747-7758.

9 Y. Sun, W. Tong, X. J. Xu and Y. H. Zhang, Possible doubleexchange interaction between manganese and chromium in LaMn $_{1-x} \mathrm{Cr}_{x} \mathrm{O}_{3}$, Phys. Rev. B: Condens. Matter Mater. Phys., 2001, 63, 174438.

10 H. Hebert, C. Martin, A. Maignan, R. Reloux, M. Hervieu, N. Ngnyen and B. Raveau, Induced ferromagnetism in $\mathrm{LaMnO}_{3}$ by Mn-site substitution: the major role of $\mathrm{Mn}$ mixed valency, Phys. Rev. B: Condens. Matter Mater. Phys., 2002, 65, 104420.

11 J. W. Cai, C. Wang, B. G. Shen, J. G. Zhao and W. S. Zhan, Colossal magnetoresistance of spin-glass perovskite $\mathrm{La}_{0.67} \mathrm{Ca}_{0.33} \mathrm{Mn}_{0.9} \mathrm{Fe}_{0.1} \mathrm{O}_{3}$, Appl. Phys. Lett., 1997, 71, 17271729.

12 X. Chen, Z. Wang, R. Li, B. Shen, W. Zhan, J. Sun, J. Chen and C. Yan, The magnetic and transport properties of Fe doped $\mathrm{La}_{0.5} \mathrm{Ca}_{0.5} \mathrm{MnO}_{3}$, J. Appl. Phys., 2000, 87, 5594-5596.

13 L. Righi, P. Gorria, M. Insausti, J. Gutierrez and J. M. Barandiaran, Influence of $\mathrm{Fe}$ in giant magnetoresistance ratio and magnetic properties of
$\mathrm{La}_{0.7} \mathrm{Ca}_{0.3} \mathrm{Mn}_{1-x} \mathrm{Fe}_{x} \mathrm{O}_{3}$ perovskite type compounds, J. Appl. Phys., 1997, 81, 5767-5769.

14 J. Gustierrez, J. M. Barandiaran, M. Insausti, L. Lezama, A. Pena, J. J. Blance and T. Rojo, Magnetic and transport properties of $\mathrm{Pb}$ perovskites and $\mathrm{Fe}$ containing giant magnetoresistance perovskites, J. Appl. Phys., 1998, 83, 7171-7173.

15 M. Pissas, G. Kallias, E. Devlin, A. Simopoulos and D. Niarchos, Mossbauer study of $\mathrm{La}_{0.75} \mathrm{Ca}_{0.25} \mathrm{Mn}_{0.98} \mathrm{Fe}_{0.02} \mathrm{O}_{3}$ compound, J. Appl. Phys., 1997, 81, 5770-5772.

16 C.-J. Liu, A. Bhaskar, H. J. Huang and F.-H. Lin, Transport properties ofBi-doped FeSe superconductor up to $700 \mathrm{~K}$, Appl. Phys. Lett., 2014, 104, 252602.

17 A. Bhaskar, J. J. Yuan and C.-J. Liu, The effect of Si doping on the thermoelectric and magnetic properties of $\mathrm{Ca}_{0.98} \mathrm{Bi}_{0.02} \mathrm{Mn}_{1-x} \mathrm{Si}_{x} \mathrm{O}_{3-\delta}(0.00,0.02$, and 0.03), Mater. Sci. Eng., B, 2014, 186, 48-53.

18 K. H. Ahn, X. W. Wu, K. Lin and C. L. Chien, Effects of Fe doping in the colossal magnetoresistive $\mathrm{La}_{1-x} \mathrm{Ca}_{x} \mathrm{MnO}_{3}, J$. Appl. Phys., 1997, 81, 5505-5507.

19 K. H. Ahn, X. W. Wu, K. Lin and C. L. Chien, Magnetic properties and colossal magnetoresistance of $\mathrm{La}(\mathrm{Ca}) \mathrm{MnO}_{3}$ materials doped with Fe, Phys. Rev. B: Condens. Matter Mater. Phys., 1996, 54, 15299-15302.

20 G. H. Jonker, Semiconducting properties of mixed crystals with perovskite structure, Physica, 1954, 20, 1118-1122.

21 T. S. Orlova, J. Y. Laval, P. Monod, J. G. Noudem, V. S. Zahvalinskii, V. S. Vikhnin and Y. P. Stepanov, Effect of Fe doping on structure, charge ordering, magnetic and transport properties of $\mathrm{La}_{0.33} \mathrm{Ca}_{0.67} \mathrm{Mn}_{1-y} \mathrm{Fe}_{y} \mathrm{O}_{3}(0 \leq y \leq$ 0.06), J. Phys.: Condens. Matter, 2006, 18, 6729-6748.

22 C. H. Chen and S.-W. Cheong, Commensurate to incommensurate charge ordering and its real-space image in $\mathrm{La}_{0.5} \mathrm{Ca}_{0.5} \mathrm{MnO}_{3}$, Phys. Rev. Lett., 1996, 76, 4042-4045.

23 I. Dhiman, A. Das, A. K. Nigam and U. Gasser, Influence of Bsite disorder in $\mathrm{La}_{0.5} \mathrm{Ca}_{0.5} \mathrm{Mn}_{1-x} \mathrm{Bi}_{y} \mathrm{O}_{3}(\mathrm{~B}=\mathrm{Fe}, \mathrm{Ru}, \mathrm{Al}$, and Ga) manganites, J. Phys.: Condens. Matter, 2011, 23, 246006.

24 G. H. Rao, J. R. Sun, A. Kattwinkel, L. Haupt, K. Barner, E. Schmit and E. Gmelin, Magnetic, electric and thermal properties of $\mathrm{La}_{0.7} \mathrm{Ca}_{0.3} \mathrm{Mn}_{1-x} \mathrm{Fe}_{x} \mathrm{O}_{3}$ compounds, Phys. B, 1999, 269, 379-385.

25 T. Holstein, Studies of polaron motion: Part I. The molecular-crystal model, Ann. Phys., 1959, 8, 325-342.

26 C. S. Lue, Y. K. Kuo, F. H. Hsu, H. H. Li, H. D. Yang, P. S. Fodor and L. E. Wenger, Thermal hysteresis in the charge-density-wave transition of $\mathrm{Lu}_{5} \mathrm{Rh}_{4} \mathrm{Si}_{10}$, Phys. Rev. B: Condens. Matter Mater. Phys., 2002, 66, 033101.

27 A. Smontara, K. Biljakovic, J. Mazuer, P. Monceau and F. Levy, Thermal hysteresis in thermopower of the chargedensity-wave system $\left(\mathrm{NbSe}_{4}\right)_{10} \mathrm{I}_{3}, J$. Phys.: Condens. Matter, 1992, 4, 3273-3281.

28 M. Jaime, M. B. Salamon, M. Rubinstein, R. E. Treece, J. S. Horwitz and D. B. Chrisey, High-temperature thermopower in $\mathrm{La}_{2 / 3} \mathrm{Ca}_{1 / 3} \mathrm{MnO}_{3}$ films: evidence for polaronic transport, Phys. Rev. B: Condens. Matter Mater. Phys., 1996, 54, 11914-11917. 
29 V. H. Crespi, L. Lu, Y. X. Jia, K. Khazeni, A. Zetll and M. L. Cohen, Thermopower of single-crystal $\mathrm{Nd}_{1-x}(\mathrm{Sr}, \mathrm{Pb})_{x} \mathrm{MnO}_{3-\delta}$, Phys. Rev. B: Condens. Matter Mater. Phys., 1999, 53, 14303-14308.

30 M. J. Gillan and D. Wolf, Point-defect diffusion from coherent quasielastic neutron scattering, Phys. Rev. Lett., 1989, 55, 1299-1302.

31 N. F. Mott and E. A. Davis, Electronic Process in Noncrystalline Materials, Oxford University Press, Oxford, 2nd edn, 1979.

32 F. Rivadulla, M. Freita-Aivite, M. A. Lopez-Quintela, L. E. Hueso, D. R. Miguens, P. Sande and J. Rivas, Coexistence of paramagnetic-charge-ordered and ferromagnetic-metallic phase in $\mathrm{La}_{0.5} \mathrm{Ca}_{0.5} \mathrm{MnO}_{3}$ evidenced by electron spin resonance, J. Appl. Phys., 2002, 91, 785-788.

33 P. Levy, L. Granja, E. Indelicato, G. Polla, D. Vega and F. Parisi, Effect of Fe doping in $\mathrm{La}_{1 / 2} \mathrm{Ca}_{1 / 2} \mathrm{MnO}_{3}$, J. Magn. Magn. Mater., 2001, 226, 794-796.

34 A. Das and P. D. Babu, Ionic size effect in charge-ordered $\mathrm{La}_{0.5} \mathrm{Ca}_{0.5} \mathrm{MnO}_{3}$, Phys. Rev. B: Condens. Matter Mater. Phys., 2004, 70, 224404.

35 S. S. Kekade, R. S. Devan, A. V. Deshmukh, D. M. Phase, R. J. Choudhary and S. I. Patil, Electrical transport behavior and charge ordering phenomena in $\mathrm{La}_{0.5} \mathrm{Ca}_{0.5} \mathrm{MnO}_{3}$, J. Alloys Compd., 2016, 682, 447-453. 